Arquero, J.; Fernández-Polvillo, C.; Michel, G.; Ramírez, A. (2018). Relaciones entre aprensión y autoeficacia comunicativa en los estudiantes de contaduría en México. Contaduría Universidad de Antioquia, 72, 39-57

Doi: https://doi.org/10.17533/udea.rc.n72a03

\title{
Relaciones entre aprensión y autoeficacia comunicativa en los estudiantes de contaduría en México*
}

\author{
José Luis Arquero* \\ arquero@us.es \\ Universidad de Sevilla \\ Carmen Fernández-Polvillo \\ carmenfp@us.es \\ Universidad de Sevilla \\ Gracia Patricia Michel Vázquez \\ graciapa@cucsur.udg.mx \\ Universidad de Guadalajara \\ Araceli Ramírez Meda \\ aramirez@cucsur.udg.mx \\ Universidad de Guadalajara
}

Los datos para este trabajo fueron obtenidos en el marco de una investigación más amplia financiada con una beca PROMEP (Programa de Mejoramiento del Profesorado. Dirección General Educación Superior Universitaria, gobierno de México) para estudios de posgrado.

** Autor para correspondencia. Dirección postal: FCEYE - Departamento de Contabilidad y Economía Financiera Ramón y Cajal nº 1, 41018 - Sevilla, España. 


\section{Relaciones entre aprensión y autoeficacia comunicativa en los estudiantes de contaduría en México}

Resumen: La contabilidad es una actividad íntimamente ligada a la comunicación. La relevancia de las capacidades comunicativas ha sido resaltada por gran número de instituciones académicas y profesionales con el apoyo de los resultados de la investigación, concluyendo que son esenciales para un adecuado desempeño de las tareas profesionales. Sin embargo, los esfuerzos educativos para su desarrollo no parecen tener éxito, debido, en parte, a la barrera que crea la aprensión comunicativa (CA). Una posible forma de aliviar esa barrera es mejorando las percepciones de autoeficacia en comunicación (CSE) de los estudiantes, dado el vínculo que existe entre autoeficacia y aprensión, constatado por la investigación. En esa línea, el propósito de este trabajo es investigar si el vínculo entre CA y CSE también está presente en estudiantes latinoamericanos de contabilidad, población en la que no se ha estudiado esta cuestión. Para ello se distribuyó en clase una batería de cuestionarios ya utilizados en la literatura a una muestra de alumnos iniciales de contabilidad de una universidad pública mexicana. Los resultados obtenidos, usando la misma metodología que los referentes internacionales arrojaron resultados muy similares de correlaciones negativas entre los puntajes CA y CSE. Por lo tanto, el patrón de relaciones no se ve afectado culturalmente, sino que está presente en estudiantes de diferentes contextos, lo que, como implicación, sugiere que las experiencias exitosas de mejora de la CSE y reducción de la CA podrían ser transferibles.

Palabras clave: Aprensión comunicativa, autoeficacia comunicativa, barreras para el desarrollo de habilidades, contabilidad, México.

Relationships between apprehension and communicative self-efficacy in accounting students in Mexico Abstract: Accounting is an activity closely linked to communication. The relevance of communicative capacities has been highlighted by a great number of academic institutions and professionals with the support of research results, concluding that they are essential for an appropriate performance in professional tasks. However, educational efforts for their development do not appear to be successful, partly due to the barrier created by communicative apprehension (CA). A possible way to alleviate such barrier is improving the students' perceptions of communicative self-efficacy (CSE), given the existing link between self-efficacy and apprehension, as evidenced by research studies. Along this line, the purpose of this work is to investigate if the link between CA and CSE is also present in Latin American accounting students, a population that has not been studied on this issue. To this end, a battery of questionnaires already used in the literature was applied to a sample of first-year accounting students of a Mexican public university. Using the same methodology as international referents, the study yielded similar results of negative correlations between CA and CSE scores. Therefore, the relationship pattern is not culturally affected, but is rather present in students of different contexts, which suggests that successful experiences of CSE improvement and CA reduction could be transferable.

Keywords: Communicative apprehension, communicative self-efficacy, skill development barriers, accounting, Mexico.

Relations entre appréhension et auto-éfficacité communicative chez les étudiants en comptabilité au Mexique Résumé: La comptabilité est une activité étroitement liée à la communication. L'importance des capacités comminicatives a été soulignée par un grand nombre d'établissements académiques et professionnels basés sur les résultats de recherche, et qui concluent que ces capacités sont essentielles pour une performance appropriée des tâches professionnelles. Néanmoins, les efforts éducatifs pour son développement n'ont pas été réussis à cause de, en quelque sorte, la barrière créée par l'appréhension communicative (AC). Une facon d'alléger cette barrière est d'améliorer les perceptions d'auto-efficacité en communication (AEC) chez les étudiants, vu le lien entre l'autoefficacité et l'appréhension, constaté par la recherche. Le propos de ce travail est donc de trouver si le lien entre l'AC et le AEC existe aussi chez les étudiants latinoaméricains de comptabilité, dont il n'y a aucun étude préalable à ce rapport. Pour ce faire, une batterie de questionnaires - qui ont déjà été utilisés en la littérature comptable- a été distribuée en cours à un groupe d'étudiants débutants en comptabilité d'une université publique mexicaine. Les résultats obtenus -en faisant appel à la même méthodologie que les referents internacionaux- ont montré des résultats très similaires de correlations negatives entre les points AC y AEC. Par conséquent, le patron de relations $n$ 'est pas affecté culturellement mais il existe chez des étudiants de différents contextes, ce qui - implicitementsugère que les expériences réussites d'amélioration de la AEC et la réduction de la AC pourraient être transférables. Mots-clés : Appréhension communicative, auto-efficacité communicative, barrières au développement des habilités, comptabilité, Mexique

\section{Relações ente apreensão e autoeficácia comunicativa nos estudantes de Contabilidade no México}

Resumo: A contabilidade é uma atividade intimamente ligada à comunicação. A relevância das capacidades comunicativas foi exaltada por um grande número de instituições acadêmicas e profissionais com o apoio dos resultados da pesquisa, concluindo que são indispensáveis para uma adequada execução das tarefas profissionais. Porém, os esforços educativos para o seu desenvolvimento parecem não ser bem sucedidos, devido, em parte, à barreira que produz a apreensão comunicativa (CA). Uma possível forma de suavizar essa barreira é melhorando as percepções de autoeficácia em comunicação (CSE) dos estudantes, levando em conta o vínculo que existe entre autoeficácia e autoapreensão, comprovado pela pesquisa. Na mesma direção, o objetivo deste trabalho é pesquisar se o vínculo entre CA e CSE também se torna presente em estudantes de contabilidade da América Latina, população em que até agora não se estudou este assunto. Com este intuito, foram distribuídas em aula varias enquetes já utilizados na literatura a uma amostra inicial de alunos de contabilidade de uma universidade pública mexicana. Os resultados obtidos, usando a mesma metodologia dos referentes internacionais, mostram resultados muito semelhantes de correlações negativas entre as pontuações CA e CSE. Por este motivo, o padrão de relações não se vê afetado culturalmente, mas está presente em estudantes de diferentes contextos, o que ocasiona que as experiências bem sucedidas da CSE e diminuição da CA possam ser transferidas.

Palavras chave: Apreensão comunicativa, autoeficácia comunicativa, barreiras para o desenvolvimento de habilidades, contabilidade, México. 


\title{
Relaciones entre aprensión y autoeficacia comunicativa en los estudiantes de contaduría en México
}

\author{
José Luis Arquero, Carmen Fernández-Polvillo, \\ Gracia Patricia Michel Vázquez y Araceli Ramírez Meda
}

Doi: https://doi.org/10.17533/udea.rc.n72a03

Primera versión recibida en mayo de 2018- Versión final aceptada en junio de 2018

\section{Introducción}

Arquero afirma que:

La contabilidad es una actividad intrínsecamente unida a la comunicación. De este modo, un profesional que desarrolle su trabajo en el área contable debe tener un nivel mínimo de capacidades comunicativas sin el cual, sencillamente, no podrá llevar a cabo su trabajo de forma adecuada. (Arquero, 2001, p.76)

Estas capacidades no son únicamente escritas, es decir, elaborar informes, sino que son muy variadas (orales y escritas) y deben ajustarse a distintos escenarios y contextos (formales, informales, usuarios, etc. (Arquero, González, Hassall y Joyce, 2013)

Esta vinculación se evidencia (Arquero, 2001) en la propia definición de contabilidad, en las declaraciones institucionales de organismos de gran peso y en el respaldo a dichas declaraciones proporcionado por los resultados de la investigación publicada.

La relevancia de éstas capacidades, junto con las interpersonales y de resolución de problemas, ha hecho que se hayan realizado numerosos intentos de desarrollo de las mismas en el currículum contable desde hace tiempo (como puede comprobarse en las revisiones de la literatura de Rebele et al., 1998a y 1998b, para experiencias tempranas de innovación). Sin embargo, estas experiencias no parecen tener el éxito generalizado que pudiese esperarse; de forma que numerosos autores (e.g. Albretch y Sack, 2000; Bui y Porter, 2010 o Howcroft, 2017, entre otros) destacan la persistencia de una brecha formativa 
en capacidades, especialmente de comunicación, que parece independiente del esfuerzo educativo. (Craig y McKinney, 2010)

Una de las barreras que parece estar impidiendo la efectividad de las intervenciones educativas es la aprensión comunicativa (AC) (Arquero, Donoso, Hassall, y Joyce, 2007) temor o incomodidad ante situaciones de comunicación que, además es prevalente entre los estudiantes y profesionales de contabilidad con independencia del área geográfica. (Hassall, Arquero, Joyce y González, 2013a)

Unas de las formas propuestas para superar la barrera que constituye la alta aprensión comunicativa es a través de su vínculo con la autoeficacia comunicativa (CSE), (Hassall et al., 2013a) por lo que el objetivo de este trabajo es investigar para una muestra de estudiantes de contaduría mexicanos las relaciones entre aprensión y autoeficacia, utilizando la metodología de Hassall et al. (2013a) con alumnos de la Sheffield Hallam University y Arquero et al. (2013) con alumnos españoles.

Este trabajo contribuye a la muy escasa literatura sobre este tema con alumnos de Latinoamérica y, en la medida en que confirma la existencia de vínculos con independencia de contexto geográfico, da pie a considerar transferibles las experiencias exitosas para la reducción de la aprensión que puedan ser efectivas para otros alumnos.

El resto del trabajo se estructura como sigue, el siguiente apartado realiza una revisión de la literatura relevante, tanto sobre capacidades como sobre aprensión y autoeficacia. En el apartado de metodología, se presentan los instrumentos usados y el procedimiento de distribución seguido de los apartados de resultados, conclusiones y referencias.

\section{Antecedentes teóricos}

\section{II.1 Importancia de las habilidades de comunicación para la profesión contable}

Arquero (2001) resalta que en las definiciones de contabilidad de mayor consenso se encuentra explícita la íntima relación entre contabilidad y comunicación, destacando la contenida en el documento ASOBAT de la AAA (American Accounting Association, 1966) que define a la contabilidad como "el proceso de identificación, medida y comunicación de la información para la formación de juicios o toma de decisiones por parte de los usuarios" (p.2). Ésta perspectiva comunicacional (sistema de información que convierte datos en información a ser comunicada a múltiples usuarios) se traslada a las normativas contables que, como en el caso de la española (influenciada por las normas internacionales) recoge en su marco conceptual los requisitos de la 
información contable, entre los que se destacan la claridad, como exigencia de la comprensibilidad. Así, siguiendo a Arquero consideramos que

La información contable no se elabora para que quede oculta, sino para ser comunicada; por lo que la incapacidad de presentar y comunicar adecuadamente esta información, en función de las características del usuario y del tipo de decisión que éste debe tomar, supone la inutilidad de todo el esfuerzo invertido en el proceso previo. (Arquero, 2001, p. 77)

Las instituciones, tanto académicas como profesionales más representativas en la contabilidad, también han destacado en sus declaraciones y posicionamientos la importancia de las capacidades de comunicación para un desempeño adecuado de las tareas de un profesional del área. Como indican Arquero, Fernández-Polvillo, Hassall y Joyce (2017), el debate sobre la relevancia de capacidades no técnicas, y la necesidad de incluirlas en la formación superior en contabilidad se inicia en Estados Unidos, especialmente a partir del Informe Bedford (AAA, 1986) en el que se indica que "muchos graduados en contabilidad no saben cómo comunicarse, son incapaces realizar razonamientos lógicos y tienen limitaciones en sus habilidades de resolución de problemas, como evidencia notoria de la necesidad de acometer un cambio radical en la formación en contabilidad" (p.98). El principal instituto de contadores públicos de EE.UU. (American Institute of Certified Public Accountants - AICPA) hace suyas las conclusiones del Informe Bedford, publicando poco después el documento Future Issues, (AICPA, 1987) en el que resalta el gap entre necesidades de la profesión y la formación ofertada en las instituciones de educación, y la revisión del Informe Albers, (AICPA, 1988) en el que actualiza sus requisitos de formación, dando una mayor relevancia conocimientos no estrictamente contables y a las capacidades no técnicas. La muestra definitiva de apoyo de la profesión a esas nuevas líneas de revisión curricular es el documento Perspectives on Education: Capabilities for Success in the Accounting Profession (Arthur Andersen y Co. et al., 1989) suscrito por todas las grandes empresas de contabilidad y auditoría, en el que explícitamente respaldan las conclusiones de la AAA y del AICPA.

A partir de ese punto, que supone una inflexión (Arquero, 2000) y la generalización de este debate, todos los posicionamientos sobre formación -desde las distintas versiones del Core Competency Framework (AICPA, 1999; 2016) hasta las últimas versiones del International Education Standard no 3: Initial Professional Development - Professional Skills (IFAC, 2017) o del Skills Framework, (Common Content Project, 2017) pasando por el modelo de currículo contable de la UNCTAD (2011) resaltan la enorme importancia de un conjunto de capacidades no técnicas (soft o transferable skills) entre las que destacan las de comunicación. 
Arquero, Fernández-Polvillo, Michel, Ramírez. Relaciones entre aprensión y autoeficacia comunicativa...

Pero ésta relevancia no se basa sólo en los enunciados institucionales. Hassall et al. (2013a) destacan que hay un consenso geográfico sobre su importancia para los futuros profesionales, reflejado en los resultados de la investigación publicada. Así, entre otros muchos, los trabajos de Christensen y Rees (2004) y Diamond (2005) en los Estados Unidos; los de Hassall, Joyce, Arquero y Donoso (2003 y 2005) y Arquero, Donoso, Hassall y Joyce (2001 y 2007) en un contexto europeo; Kavanagh y Drennan (2008), De Lange, Jackling y Gut. (2006), Jackling y De Lange (2009) desde una perspectiva australiana o Wells, Gerbic, Kranenburg y Bygrave (2009), Gray (2010), Gray y Murray (2011) en Nueva Zelanda, aportan evidencias empíricas de la importancia otorgada a las capacidades, especialmente las comunicativas, por distintos grupos de interés (profesionales y empleadores, académicos, etc.).

A pesar de que ésta importancia es conocida, Arquero et al. (2017) destacan que también existe consenso entre empleadores y académicos en que, en muchos casos, el nivel que presentan los titulados en las habilidades y cualidades personales requeridas no es el adecuado. (ver por ejemplo Albrecht y Sack, 2000; Graham, Hampton, y Willett, 2009; Quible y Griffin, 2007 o Jackling y De Lange, 2009) Como consecuencias no deseadas, las carreras profesionales se ven perjudicadas por deficiencias en habilidades de comunicación (Christensen y Rees, 2004).

Ésta brecha entre las habilidades requeridas y las exhibidas no es el resultado de la falta de esfuerzo para desarrollar esas competencias $^{1}$, sino que parece existir independientemente del esfuerzo de los profesionales académicos (v.g. Craig y McKinney, 2010; Kerby y Romine, 2009; Stoner y Milner, 2010 o Sharifi, McCombs, Fraser, y McCabe, 2009) apuntando a la existencia de trabas o barreras a estos esfuerzos que limitan su eficacia. (Hassall et al., 2013a).

\section{II.2 Barreras para el desarrollo de la comunicación: aprensión de la comunicación}

Stanga y Ladd (1990) y Ruchala y Hill (1994) destacan que, a pesar de la importancia de las habilidades de comunicación, se sabe relativamente poco sobre los obstáculos que enfrentan los estudiantes de contabilidad, y el profesorado, cuando intentan desarrollarlas. Hassall, Arquero, Joyce y González (2013b) apuntan a la ansiedad, que puede en muchos casos, impedir un desempeño adecuado y, con el tiempo, incluso crear una barrera que bloquea la posibilidad de desarrollos futuros. Así, uno de los principales obstáculos

1 Esto es fácilmente constatable a través de las revisiones y clasificación de los trabajos publicados en las principales revistas de docencia de la contabilidad realizadas por Apostolou, Dorminey, Hassell y Rebele. $(2015 ; 2016 ; 2017)$ 
para los estudiantes de contabilidad puede ser la aprensión comunicativa (CA). (Arquero et al., 2007)

McCroskey (1984) define la aprensión de la comunicación como "el nivel de miedo y ansiedad de un individuo asociado con la comunicación real o anticipada con otra persona" (p. 78). Esto sugiere (Hassall et al., 2013b) que los individuos aprensivos son menos capaces de comunicarse de manera efectiva, por lo que estos efectos pueden influir en la educación, en los procesos de reclutamiento y la carrera profesional futura. En etapas más tempranas, Arquero, Fernández-Polvillo y Valladares (2017) demuestran que el nivel de CA influye incluso en la elección de si continuar estudiando en niveles superiores y qué estudiar.

Como educadores en instituciones universitarias, donde la empleabilidad de nuestros estudiantes es esencial, los efectos de CA en el desarrollo de las competencias requeridas y el posterior éxito de nuestros estudiantes como futuros profesionales son de la mayor importancia. Siguiendo a Hassall et al. (2013b), en un sistema educativo más tradicional basado en un enfoque expositivo y centrado en la figura del profesor, la CA puede no ser un problema significativo; sin embargo con metodologías más activas (v.g. donde se requiere la participación voluntaria del estudiante, como el trabajo en grupo) la CA es un factor que puede influir negativamente en la actitud de los individuos (McCroskey y Anderson, 1976) y, posteriormente, afectar los resultados en las evaluaciones.

Los peores resultados de los aprensivos no parece que se deban a la relación CA- inteligencia: McCroskey, Daly, Richmond y Cox (1976), usando una amplia gama de medidas de personalidad e inteligencia, no encontraron evidencia de relación negativa entre ambas, pero informaron que altos niveles de CA estaban asociados con comportamientos de evasión (como estar sentados en la parte posterior de las aulas, elegir optativas que no requieren participación/ interacción, o no buscar la asistencia de un tutor), que finalmente podrían afectar el reconocimiento del progreso y las necesidades del estudiante y pueden perjudicar el rendimiento educativo. (Fordham y Gabbin, 1996)

El problema para el área contable de la $C A$ es que, pese a que la comunicación es una habilidad clave en contabilidad, muchos estudios destacan que los estudiantes de contabilidad tienden a presentar (I) niveles más bajos que los requeridos en habilidades de comunicación y (II) niveles más altos de CA (una barrera potencial para el desarrollo de habilidades de comunicación) en comparación con otros estudiantes.

Los estudios iniciales, con estudiantes de EE.UU., concluyeron que los estudiantes que optaban por la contabilidad como carrera tenían niveles de CA oral superiores a la media (v.g. Stanga y Ladd, 1990), resultados confirmados agregando medidas de CA escrita (Simons, Higgins, y Lowe, 1995). Estudios 
Arquero, Fernández-Polvillo, Michel, Ramírez. Relaciones entre aprensión y autoeficacia comunicativa...

posteriores realizados en otros países arrojaron resultados similares, concluyendo que los estudiantes de contabilidad tienden a presentar mayores niveles de CA que los estudiantes matriculados en grados cercanos: así para Reino Unido y España: Hassall, Joyce, Ottewill, Arquero y Donoso (2000), Arquero et al. (2007); para Irlanda: Byrne, Flood y Shanahan (2009); para Nueva Zelanda: Gardner, Milne, Stringer y Withing (2005) o Canadá: Aly and Islam (2003). Por lo tanto, aunque se podrían introducir esfuerzos para desarrollar habilidades de comunicación para aquellos estudiantes con niveles más altos de $\mathrm{CA}$, esas estrategias podrían resultar ineficaces y la mejora en tales habilidades no ocurriría. (Spitzberg y Cupach, 1984; Allen y Bourhis, 1996)

En esta línea, Hassall et al. (2013b) señalan que desafortunadamente los cursos de contabilidad parecen tener un impacto mínimo en la reducción de los niveles de AC, tal como lo sugieren los estudios de Hassall et al. (2000), Aly y Islam (2003) y Gardner et al. (2005) en contextos anglosajones, o Michel, Ramírez, Arquero y Fernández-Polvillo (2015) para estudiantes mexicanos. En general estos estudios encontraron poco o ningún cambio en los niveles de CA a medida que los estudiantes avanzaban a través de cursos de contabilidad.

\section{II.3 Comunicación Autoeficacia y enlaces con CA}

Hassall et al. (2013a) estudiaron el vínculo entre AC y autoeficacia de comunicación (CSE) sugiriendo que la existencia de tal relación podría proporcionar "un posible desarrollo en términos de entender la barrera para el desarrollo de habilidades de comunicación y el potencial para redireccionar esfuerzos para aliviar y eliminar la barrera." (p.178)

El razonamiento se basa en la definición de autoeficacia y cómo se desarrolla. La autoeficacia se define (Bandura, 1997) como el nivel de confianza que tienen las personas en su capacidad para ejecutar cursos de acción o alcanzar resultados de desempeño específicos. Por lo tanto, siguiendo a Hassall et al. (2013a) la autoeficacia es la creencia del individuo en su capacidad para lograr un resultado específico; independiente del requisito de habilidad real. Entonces, si la autoeficacia (creencia) del individuo está sustancialmente por debajo de la habilidad requerida (o la percepción del nivel requerido), entonces esto se convertirá en una barrera importante para el desarrollo en esa área contextual; y podríamos agregar que, si la diferencia entre la autoeficacia percibida y el nivel requerido percibido para una tarea es demasiado alta, entonces es probable que aparezcan comportamientos de evasión y ansiedad. En tareas que involucran comunicación esto se corresponde con la CA.

La hipótesis de Hassall et al. (2013a) es que, si las intervenciones educativas pueden mejorar la autoeficacia de comunicación de los estudiantes, entonces es probable que las actividades futuras relacionadas con la comunicación se 
perciban como menos "temibles", más fáciles de lograr y la CA se reducirá, eliminando la barrera: así,

Si un individuo cree que tendrá éxito, estará más inclinado a intentar una tarea, se esforzará más para completar la tarea y estará preparado para mantener este esfuerzo por un período de tiempo más largo a pesar de encontrar obstáculos. Los individuos intentarán tareas en las que tengan una alta autoeficacia y evitarán tareas en las que tengan baja autoeficacia. (Hassall et al., 2013a, p.164)

Los resultados de todos los estudios que relacionan CA con las medidas de CSE indican que existe un vínculo sólido tanto en el área contable: Hassall et al (2013a) con estudiantes de la Sheffield Hallam University o Arquero et al. (2013) con estudiantes españoles; como con estudiantes de otras áreas (McCroskey, Burroughs, Daun, y Richmond, 1990 y Teven, Richmond, McCroskey, y McCroskey, 2010) en muestras de EE. UU.

\section{Objetivo y metodología}

\section{III.1 Objetivo y pregunta de investigación}

El objetivo principal de este documento es replicar a Hassall et al. (2013a) al estudiar una población donde el impacto de CA no ha sido estudiado hasta la fecha: estudiantes latinoamericanos, en concreto, mexicanos. La razón es que, como se indicó anteriormente, las intervenciones educativas basadas en la mejora de la CSE podrían ayudar a eliminar la barrera formada por niveles más altos de CA y por lo tanto, ayudar a desarrollar las habilidades de comunicación esenciales. Sin embargo, esas intervenciones podrían ser transferibles si el enlace CA- CSE también está presente en los estudiantes con independencia del contexto. Por lo tanto, la principal pregunta de investigación de este estudio es:

RQ1: ¿Qué asociación existe entre los niveles de aprensión comunicativa y las creencias de autoeficacia comunicativa en los estudiantes mexicanos?

Dado que la presente investigación utiliza los mismos instrumentos que otras investigaciones para estudiantes con diferentes antecedentes culturales (Hassall et al. 2013 y Arquero et al., 2013), se formula una segunda pregunta de investigación.

RQ2: ¿Los patrones de relaciones son similares a los encontrados para otras culturas?

\section{III.2 Instrumentos}

\section{III.2.1 Aprensión comunicativa}

$\mathrm{Al}$ igual que en estudios previos centrados en CA (Arquero et al, 2013 y 2017; Hassall et al, 2013a), el instrumento constaba de dos cuestionarios para medir CA a contestar por los estudiantes. 
Arquero, Fernández-Polvillo, Michel, Ramírez. Relaciones entre aprensión y autoeficacia comunicativa...

El primer cuestionario mide la aprensión comunicativa oral usando la versión en español (Arquero et al, 2013) del Personal Report of Communication Apprehension (PRCA-24) desarrollado por McCroskey $(1984,2006)$ con las adaptaciones a muestras universitarias introducidas por Hassall et al. (2000) ${ }^{2}$. El PRCA-24 consta de 24 ítems sobre comunicación en cuatro contextos: entornos formales (representados y explicados como situaciones de entrevista y presentación) y entornos informales (representados y explicados como situaciones de conversación y discusión grupal).

El segundo instrumento utilizado, para medir la aprensión comunicativa escrita, es la versión en español (Arquero et al., 2013) del WCA-6 (Arquero, Fernández, Hassall y Joyce, 2012). El WCA-6 es una versión reducida (6 ítems) del WCA-24 (Hassall et al., 2000), una adaptación a estudiantes universitarios de la prueba de aprensión escrita WAT. (Daly y Miller, 1975)

Las declaraciones en ambos cuestionarios deben responderse en una escala tipo Likert de 5 puntos, que van desde "Muy en desacuerdo" a "Muy de acuerdo" y una puntuación más alta indica un nivel más alto de CA.

El cuestionario utilizado para medir la autoeficacia de la comunicación fue la versión en español (Arquero et al., 2013) del instrumento desarrollado por Hassall et al. (2013a). Este cuestionario que consta de un total de 32 ítems, se desarrolló siguiendo las pautas establecidas por Bandura (2006) y se diseñó para medir dos constructos: la autoeficacia de la comunicación oral y la autoeficacia de la comunicación escrita. La autoeficacia de la comunicación oral y la autoeficacia de la comunicación escrita se dividieron en dos subsecciones: comunicación en diferentes contextos o tareas, y aspectos técnicos de las habilidades de comunicación.

Se les pide a los estudiantes que respondan a la siguiente pregunta general: ¿Qué tan seguro está de que podría hacer lo siguiente? Califique su nivel de confianza utilizando una escala que va desde 0 (sin confianza en absoluto) a 10 (completamente seguro). Ejemplos de artículos para CSE escritos son los siguientes: "Escribir un ensayo de una o dos páginas en respuesta a una pregunta determinada" (WCSE, contexto) o "Escribir oraciones simples con buena gramática" (WCSE, aspectos técnicos). Para la comunicación oral, dos puntos representativos son: "Una entrevista individual formal con un empleador" para las tareas contextuales, o "Preparar una presentación bien organizada y secuenciada con una buena introducción, cuerpo y conclusión" para los aspectos técnicos. Los puntajes más altos son indicativos de niveles más altos de autoeficacia percibida.

2 Ésta adaptación ha sido utilizada en varios trabajos de investigación, por ejemplo, Arquero et al. (2007 y 2017); Gardner et al. (2005); Joyce, Hassall, Arquero y Donoso (2006) en inglés y Arquero et al. (2013) en español, formando un sólido y confiable conjunto de resultados. 


\section{III.2.2 Muestra y procedimiento}

Para este estudio se contó con una muestra de conveniencia de estudiantes matriculados en el grado de contabilidad de una universidad pública mexicana. Se consideró relevante verificar la relación CA y de CSE en los estudiantes previo a cualquier experiencia en la Universidad, por lo que la muestra estuvo compuesta por alumnos matriculados en el primer curso aplicándose el instrumento al inicio del cuatrimestre distribuyéndose el instrumento en tiempo de clase.

Para evitar sesgos en las respuestas, un miembro del equipo de investigación explicó que el propósito del cuestionario era solo obtener datos agregados para la investigación, en modo alguno individuales, asegurando la confidencialidad de las respuestas, resaltando que no existen respuestas correctas o incorrectas, pidiendo respuestas sinceras.

Un total de 100 cuestionarios completos se reunieron e introdujeron en el SPSS para el análisis estadístico. Los estudiantes en la muestra eran en su mayoría de mujeres (60\%), la edad promedio de los estudiantes es 19.5 (DE: 3.2). Además, el $69 \%$ de los encuestados indicó tener una formación previa científica académica (frente al 31\% de literatura y arte).

\section{Resultados}

Las correlaciones entre CA escrita y la autoeficacia en la comunicación escrita son como en estudios previos significativamente negativos (tabla 1). La aprensión parece estar más relacionada con las creencias de autoconfianza sobre aspectos técnicos (coef.: -.582) que, con las diferentes situaciones o contextos propuestos, aunque sigue siendo alta (coef.: -.496). Ambos aspectos de CSE están positivamente correlacionados (coef.: .694).

Hassall et al. (2013a) resaltaron la necesidad y la oportunidad de que los estudios comparativos establezcan la medida en que los hallazgos son generalizables para los estudiantes de contabilidad de diferentes orígenes culturales. Por lo tanto, junto con nuestros propios resultados, presentamos los resultados obtenidos con los mismos instrumentos en muestras de estudiantes en diferentes contextos culturales: Hassall et al. (2013a) para estudiantes asiáticos matriculados en una universidad del Reino Unido y Arquero et al. (2013) para estudiantes de España.

Como se puede deducir de la tabla 1, los patrones de las relaciones entre CA escrita y CSE son bastante similares con independencia del contexto cultural. 
Arquero, Fernández-Polvillo, Michel, Ramírez. Relaciones entre aprensión y autoeficacia comunicativa...

Tabla 1. Relación entre AC escrita y autoeficacia

\begin{tabular}{llcccccc}
\hline \multirow{2}{*}{$\begin{array}{c}\text { WSCE } \\
\text { contexto }\end{array}$} & \multicolumn{2}{c}{$\begin{array}{c}\text { Estudio actual } \\
\text { Resultados (n:100) }\end{array}$} & \multicolumn{2}{c}{$\begin{array}{c}\text { Hassall et al. (2013a) } \\
\text { Resultados (n:228) }\end{array}$} & \multicolumn{2}{c}{$\begin{array}{c}\text { Arquero et al. (2013) } \\
\text { Resultados (n:449)* }\end{array}$} \\
\cline { 3 - 8 } & & $\begin{array}{c}\text { WSCE } \\
\text { técnica }\end{array}$ & $\begin{array}{c}\text { WSCE } \\
\text { contexto }\end{array}$ & $\begin{array}{c}\text { WSCE } \\
\text { técnica }\end{array}$ & $\begin{array}{c}\text { WSCE } \\
\text { contexto }\end{array}$ & $\begin{array}{c}\text { WSCE } \\
\text { técnica }\end{array}$ \\
\hline \multirow{2}{*}{ WCA } & Coef. corr. Pearson & -.496 & -.582 & -.475 & -.518 & -.464 & -.531 \\
& Sig. (2 colas) & .000 & .000 & .000 & .000 & .000 & .000 \\
\multirow{2}{*}{ WSCE } & Coef. corr. Pearson & & .694 & & .520 & & .520 \\
contexto & Sig. (2 colas) & & .000 & & .000 & & .000 \\
\hline
\end{tabular}

*Nota: la muestra incluye estudiantes matriculados en carreras de contabilidad, negocios, mercadotecnia y economía.

Fuente: elaboración propia a partir de resultados propios y los de Hassall et al. (2013a) y Arquero et al. (2013).

Con respecto a la comunicación oral, en la tabla 2 se presentan las correlaciones entre los puntajes CA orales para los agregados y el CSE oral. Todas las correlaciones CA-CSE son negativas a un nivel significativo. Los vínculos más fuertes son, una vez más, entre las creencias de autoeficacia en los aspectos técnicos de la comunicación (correlación CA oral- OCSE técnico: -.641), en este más fuertemente relacionado con CA en situaciones formales (coef.: -.591). Para CSE oral, la relación entre los puntajes es incluso mayor que para escrita CSE (coef.: .791).

Tabla 2. Enlaces entre CA oral (puntajes agregados) y autoeficacia

\begin{tabular}{llcccccc}
\hline & & \multicolumn{2}{c}{$\begin{array}{c}\text { Estudio actual } \\
\text { resultados (n:100) }\end{array}$} & $\begin{array}{c}\text { Hassall et al. (2013a) } \\
\text { resultados (n:228) }\end{array}$ & $\begin{array}{c}\text { Arquero et al. (2013) } \\
\text { resultados (n:449)* }\end{array}$ \\
\cline { 3 - 8 } & & OCSE & OCSE & OCSE & OCSE & OCSE & \\
& & técnica & contexto & técnica & contexto & técnica & \\
\hline CA & Coef. corr. Pearson & -.601 & -.641 & -.641 & -.588 & n.d. & n.d. \\
oral & Sig. (2 colas) & .000 & .000 & .000 & .000 & & \\
CA & Coef. corr. Pearson & -.529 & -.591 & -.623 & -.540 & -.633 & -.625 \\
formal & Sig. (2 colas) & .000 & .000 & .000 & .000 & .000 & .000 \\
CA & Coef. corr. Pearson & -.541 & -.551 & -.502 & -.496 & -.501 & -.484 \\
informal & Sig. (2 colas) & .000 & .000 & .000 & .000 & .000 & .000 \\
OCSE & Coef. corr. Pearson & & .791 & & .708 & & .798 \\
contexto & Sig. (2 colas) & & .000 & & .000 & & .000 \\
\hline
\end{tabular}

*Nota: la muestra incluye estudiantes matriculados en carreras de contabilidad, negocios, mercadotecnia y economía.

Fuente: elaboración propia a partir de resultados propios y los de Hassall et al. (2013a) y Arquero et al. (2013). 
De nuevo, comparando el resultado del presente estudio con los obtenidos por Hassall et al. (2013a) y Arquero et al. (2013), el nivel de correlaciones y los patrones son muy similares. Se puede observar que, para los estudiantes asiáticos y españoles, el vínculo más fuerte aparece entre los constructos de CA y los aspectos contextuales de competencia, mientras que para los estudiantes mexicanos el vínculo es ligeramente más fuerte entre CA y los aspectos técnicos de la comunicación.

La tabla 3 presenta las correlaciones entre los puntajes básicos de CA y la autoeficacia. La correlación negativa más alta, como cabría esperar, es entre CA en una configuración de presentación y CSE, ligeramente más alta para CSE en aspectos técnicos (coef.: -.576). Este resultado (el coeficiente de correlación más alto entre la presentación y los puntajes del CSE) coincide con los resultados de Hassall et al. (2013a), sin embargo, para los estudiantes de ese estudio, el enlace CA-CSE contextual es más fuerte (coef.: -.617) que para los estudiantes mexicanos. El estudio de Arquero et al. (2013) no proporcionó resultados a este nivel de detalle.

Por lo tanto, nuestros resultados permiten responder a las preguntas de investigación planteadas: existen relaciones significativas de moderadas a fuertes entre los puntajes de CA y las creencias de autoeficacia de comunicación en nuestra muestra de estudiantes mexicanos (RQ1). Los patrones y la fortaleza de estas relaciones son similares a los informados en otros países (RQ2), por ejemplo, por Hassall et al. (2013a) y Arquero et al. (2013). Aunque se pueden encontrar algunas diferencias menores, en términos generales, el enlace CA-CSE es comparable independientemente del contexto cultural de los estudiantes.

Tabla 3. Relaciones entre CA oral (puntajes básicos) - autoeficacia

\begin{tabular}{|c|c|c|c|c|c|}
\hline & & \multicolumn{2}{|c|}{$\begin{array}{l}\text { Estudio actual } \\
\text { resultados (n:100) }\end{array}$} & \multicolumn{2}{|c|}{ Hassall et al. (2013a) resultados ( $n: 228)$} \\
\hline & & $\begin{array}{c}\text { OCSE } \\
\text { context }\end{array}$ & $\begin{array}{c}\text { OCSE } \\
\text { tech }\end{array}$ & $\begin{array}{c}\text { OCSE } \\
\text { context }\end{array}$ & $\begin{array}{l}\text { OCSE } \\
\text { tech }\end{array}$ \\
\hline \multirow[t]{2}{*}{ Entrevista } & Coef. corr. Pearson & -.422 & -.493 & -.475 & -.372 \\
\hline & Sig. ( 2 colas) & .000 & .000 & .000 & .000 \\
\hline \multirow[t]{2}{*}{ Presentación } & Coef. corr. Pearson & -.538 & -.576 & -.617 & -.569 \\
\hline & Sig. ( 2 colas $)$ & .000 & .000 & .000 & .000 \\
\hline \multirow[t]{2}{*}{ Grupo } & Coef. corr. Pearson & -.421 & -.453 & -.474 & -.444 \\
\hline & Sig. ( 2 colas $)$ & .000 & .000 & .000 & .000 \\
\hline \multirow[t]{2}{*}{ Conversación } & Coef. corr. Pearson & -.489 & -.474 & -.418 & -.438 \\
\hline & Sig. (2 colas) & .000 & .000 & .000 & .000 \\
\hline
\end{tabular}

Fuente: elaboración propia a partir de resultados propios y los de Hassall et al. (2013a). 
Arquero, Fernández-Polvillo, Michel, Ramírez. Relaciones entre aprensión y autoeficacia comunicativa...

\section{Discusión}

El presente estudio tuvo como objetivo estudiar si en una muestra de estudiantes latinoamericanos de contabilidad existía el vínculo entre CA y la autoeficacia hallado en estudiantes en otras áreas geográficas. Con base en los hallazgos previos para otros países, se podría esperar que un individuo con altos niveles de CA muestre bajos niveles de autoeficacia de comunicación y, por lo tanto, se podría encontrar una correlación negativa entre los puntajes de CA y CSE. Sin embargo, Marambe, Vermunt y Boshuizen. (2011) encontraron que ciertos patrones de aprendizaje estaban presentes sólo en estudiantes en ciertos contextos culturales, señalando la relevancia y oportunidad de los estudios comparativos interculturales. (Hassall et al., 2013a)

Nuestros resultados, con una muestra de estudiantes mexicanos, respaldan la existencia de la relación entre CA y CSE. Los patrones de correlación, así como la fuerza e importancia de tales relaciones son muy similares a los encontrados por Arquero et al. (2013) para estudiantes españoles (que comparten ciertas referencias culturales del lenguaje) y Hassall et al (2013a) para estudiantes matriculados en una universidad británica (aunque de origen asiático, muy diferente en términos de antecedentes culturales). Estos resultados sugieren que este vínculo puede ser generalizable y apunta, por tanto, a una posible transferibilidad de los resultados de las acciones educativas efectivas para eliminar la barrera al desarrollo de habilidades de comunicación (CA).

En esta línea, los resultados de Dwyer y Fus (2002) sugieren que la aprensión podría reducirse a medida que las acciones formativas en la comunicación aumenten la autoeficacia de la comunicación. Hassall y otros (2013a), siguiendo a Bandura (1986), destacan la importancia del aprendizaje enactivo como fuente de autoeficacia. Sin embargo, las condiciones requeridas para tales experiencias: situaciones reales (no simulaciones), experiencia directa de sensación de éxito y contribución al logro de un objetivo, dificultan la inclusión de estas experiencias en la educación formal (aparte de la posibilidad de utilizar períodos de pasantías).

Aunque es más débil, la experiencia vicaria también es una fuente importante de autoeficacia. (Hassall et al., 2013a) Aquí es donde un individuo observa que un compañero tiene éxito en una tarea que, a su vez, puede fortalecer la creencia del individuo en sus propias habilidades. Dwyer y Fus (2002) se refieren a esta línea de acción que llama al uso de situaciones de comunicación de modelo de pares (discursos en su caso). Dwer y Fus proponen, siguiendo a Schunk, Hanson, y Cox, (1987) y Schunk (1995), que ver al menos tres discursos modelo (por encima del promedio, promedio y por debajo del 
promedio) aumenta la probabilidad de que los estudiantes se vean más capaces que al menos uno de los modelos y por lo tanto aumenta la autoeficacia.

Finalmente, prestando atención a un diseño cuidadoso de las tareas y situaciones donde las habilidades de comunicación deben ser desarrolladas (y evaluadas) también es señalado por diferentes autores (por ejemplo, Arquero, 2001, Simons et al 1995 o Stajkovic y Luthans, 1998). En términos generales, estos autores proponen sugerencias que eliminan las dificultades innecesarias y un aumento gradual en la complejidad de las tareas y en el impacto de la evaluación de los aspectos comunicacionales en los grados. Por lo tanto, las actividades iniciales, menos complejas, donde el aspecto comunicacional no tiene impacto en las calificaciones, podrían ayudar a los estudiantes a mejorar autoeficacia comunicativa.

\section{Referencias Bibliográficas}

Albrecht, W. S., y Sack, R. J. (2000). Accounting education: Charting the course through a perilous future. Accounting education series (Vol. 16) Sarasota, FL: American Accounting Association.

Allen, M., y Bourhis, J. (1996). The relationship of communication apprehension to communication behaviour: A meta-analysis. Communication Quarterly, 44(2), (pp. 214-226)

Aly, I. M., y Islam, M. (2003). Audit of accounting program on oral communications apprehension: A comparative study among accounting students. Managerial Auditing Journal, 18(9), (pp. 751-760)

American Accounting Association (1966). A statement of basic accounting theory. AAA. Sarassota.

American Accounting Association Committee on the Future, Content, and Scope of Accounting Education. (1986). Future of accounting education: Preparation for the expanding profession (The Bedford report). Issues in Accounting Education, 1(1), (pp. 169-195)

American Institute of Certified Public Accountants - AICPA. (1999). The AICPA Core Competency Framework for Entry into the Accounting Profession. New York, NY: AICPA.

American Institute of Certified Public Accountants - AICPA. (2016). Core Competency framework. Recuperado de https://www.aicpa.org/interestareas/accountingeducation/ resources/pages/corecompetency.aspx

American Institute of Certified Public Accountants, Education Executive Committee. (1988). Education Requirements for Entry into the Accounting Profession (Revisión del Informe del Comité Albers). New York, AICPA.

American Institute of Certified Public Accountants, Future Issues Committee. (1987). On achieving changes in Accounting Education. New York, AICPA.

Apostolou, B., Dorminey, J. W., Hassell, J. M., y Rebele, J. E. (2015). Accounting education literature review (2013-2014). Journal of Accounting Education, 33(2), 69-127. DOI: 10.1016/j.jaccedu.2015.04.001 
Arquero, Fernández-Polvillo, Michel, Ramírez. Relaciones entre aprensión y autoeficacia comunicativa...

Apostolou, B., Dorminey, J. W., Hassell, J. M., y Rebele, J. E. (2016). Accounting education literature review (2015). Journal of Accounting Education, 35(2), 20-55. DOI: 10.1016/j. jaccedu.2016.03.002

Apostolou, B., Dorminey, J. W., Hassell, J. M., y Rebele, J. E. (2017). Accounting education literature review (2016). Journal of Accounting Education, 39(2), 1-31. DOI: 10.1016/j. jaccedu.2017.03.001

Arquero, J. L. (2000). Capacidades no Técnicas en el Perfil Profesional en Contabilidad: las Opiniones de Docentes y Profesionales. Revista Española de Financiación y Contabilidad. 103, (pp. 149-172) Recuperado de https://www.jstor.org/stable/42781362

Arquero, J. L. (2001). Comunicación y Contabilidad: Implicaciones y propuestas para la formación en contabilidad. Revista de Contabilidad. 4-7, (pp.75- 99)

Arquero, J. L., Fernández-Polvillo, C., y Valladares-García, D. (2017). Communication apprehension and students' educational choices: An exploratory analysis of Spanish secondary education students. Education + Training, 59(9), (pp. 946-956)

Arquero, J. L., Fernández-Polvillo, C., Hassall, T., y Joyce, J. (2012). Properties of a short measure for written communication apprehension in university students. Madrid: Paper presented at ICERI 2012 conference.

Arquero, J. L., Fernández-Polvillo, C., Hassall, T., y Joyce, J. (2017). Relationships between communication apprehension, ambiguity tolerance and learning styles in accounting students. Revista de Contabilidad. 20(1), (pp. 13-24) Recuperado de http://dx.doi.org/10.1016/j. rcsar.2015.10.002

Arquero, J. L., González-González, J. M., Hassall, T., y Joyce, J. (2013). Autoconcepto en capacidades de comunicación y aprensión: un estudio comparativo de los estudiantes en los nuevos grados del área de Ciencias Económicas y Empresariales. Revista de Contabilidad, 16(1), (pp. 24-31)

Arquero, J.L., Donoso, J.A., Hassall, T. y Joyce, J. (2001). Vocational skills in the accounting professional profile: The Chartered Institute of Management Accountants (CIMA) employers' opinion, Accounting Education: An International Journal, 10(3), (pp. 299-313)

Arquero, J.L., Donoso, J.A., Hassall, T. y Joyce, J. (2007). Accounting students and accounting apprehension: a study of Spanish and UK students. European Accounting Review, 16 (2), (pp. 299-322)

Arthur Andersen y Co., Arthur Young, Coopers y Lybrand, Deloitte Haskins y Sells, Ernst y Whinney, Peat Marwick Main y Co., Price Waterhouse, y Touche Ross (1989). Perspectives on Education: Capabilities for Success in the Accounting Profession. (White Paper). New York: Arthur Andersen \& Co.

Bandura, A. (1997), Self-Efficacy: The Exercise of Control. W.H. Freeman, New York, NY.

Bandura, A. (2006). Guide for constructing self-efficacy scales, in Bandura, A. (Ed.), Self-Efficacy Beliefs of Adolescents. Information Age Publishing, Greenwich, CT, pp. 307-337.

Bui, B.,yPorter, B.(2010). The expectation-performancegap in accountingeducation:Anexploratory study. Accounting Education, 19(1-2), 23-50. DOI: 10.1080/09639280902875556

Byrne, M., Flood, B. \& Shanahan, D. (2009). Communication apprehension among business and accounting students in Ireland, Irish Accounting Review, 16(2), (pp. 1-19) 
Christensen, D., y Rees, D. (2004). Communication skills needed by entry-level accountants. The CPA Letter. Recuperado de www.AICPA. org/pubs/cpaltr/oct2002/AUDIT/audit2. htm (abierto el 17 de noviembre de 2004).

Common Content Project. (2017). Skills Framework. Recuperado de www.commoncontent. com

Craig, R. y McKinney, C.N. (2010). A successful competency-based writing skills development programme: results of an experiment. Accounting Education: An International Journal, 19 (3), (pp. 257-278)

Daly, J. A., y Miller, M. D. (1975). Further studies on writing apprehension: SAT scores, success expectations, willingness to take advanced courses and sex differences. Research in the teaching of English, 9, (pp. 249-253)

De Lange, P., Jackling, B. y Gut, A.M. (2006). Accounting graduates' perceptions of skills emphasis in undergraduate courses: an investigation from two Victorian universities. Accounting and Finance, 46 (3), (pp. 365-386)

Diamond, M. (2005). Accounting education, research and practice: After Enron, where do we go? European Accounting Review, 14(2), (pp. 353-362)

Dwyer, K. K., y Fus, D. A. (2002). Perceptions of Communication Competence, Self-Efficacy, And Trait Communication Apprehension: Is There an Impact on Basic Course Success? Communication Research Reports, 19(1), (pp. 29-37)

Fordham, D.R. and Gabbin, A.L. (1996). Skills versus apprehension: empirical evidence on oral communication. Business Communication Quarterly, 59(3), (pp. 88-98)

Gardner, C.T., Milne, M.J., Stringer, C.P. y Whiting, R.H. (2005). Oral and written communication apprehension in accounting students: curriculum impacts on academic performance. Accounting Education: An International Journal, 14(3), (pp. 313-36)

Graham, A., Hampton, M. \& Willett, C. (2009). What not to write: an intervention in written communication skills for accounting students. International Journal of Management Education, 8 (2), (pp. 67-74)

Gray, F.E. (2010). Specific oral communication skills desired in new accountancy graduates. Business Communication Quarterly, 73 (1), (pp. 40-67)

Gray, F.E. y Murray, N. (2011). A distinguishing factor: oral communication skills in new accountancy graduates. Accounting Education: An International Journal, 20 (3), (pp. 274-294)

Hassall, T., Arquero, J. L., Joyce, J., y Gonzalez, J. M. (2013a). Communication apprehension and communication self-efficacy in accounting students. Asian Review of Accounting, 21(2), (pp. 160-175)

Hassall, T., Arquero, J.L., Joyce, J. y González, J.M. (2013b). Communication Apprehension and Accounting Education. In Lisa Jack, Jane Davison and Russell Craig (Eds.), The Routledge Companion to Accounting Communication, Chapter 11, (pp. 166-176) Routledge. Hassall, T., Joyce, J., Arquero, J.L. y Donoso Anes, J.A. (2003). The vocational skills gap for management accountants: the Kstakeholders' perspectives. Innovations in Education and Teaching International, 40(1), (pp. 78-88) 
Arquero, Fernández-Polvillo, Michel, Ramírez. Relaciones entre aprensión y autoeficacia comunicativa...

Hassall, T., Joyce, J., Arquero, J.L. y Donoso Anes, J.A. (2005). Priorities for the development of vocational skills in management accountants: a European perspective. Accounting Forum, 29 (4), (pp. 379-394)

Hassall, T., Joyce, J., Ottewill, R., Arquero, J.L. y Donoso, J.A. (2000) 'Communication apprehension in UK and Spanish business and accounting students'. Education and Training, 42, 2: 93-100.

Howcroft, D. (2017). Graduates' vocational skills for the management accountancy profession: exploring the accounting education expectation-performance gap. Accounting Education, 26(5-6), 459-481. DOI: 10.1080/09639284.2017.1361846

International Federation of Accountants (2017). Handbook of International Education Pronouncements 2017 Edition. New York, NY: IFAC. Recuperado de https://www.ifac. org/publications-resources/2017-handbook-international-education-pronouncements

Jackling, B. y De Lange, P. (2009). Do accounting graduates' skills meet the expectations of employers? A matter of convergence or divergence. Accounting Education: An International Journal, 18 (4-5), (pp. 369-385)

Joyce, J., Hassall, T., Arquero, J. L., y Donoso, J. A. (2006). Communication apprehension and maths anxiety as barriers to communication and numeracy skills development in accounting and business education. Education and Training, 48(6), (pp. 454-464)

Kavanagh, M.H. y Drennan, L. (2008). What skills and attributes does an accounting graduate need? Evidence from student perceptions and employer expectations. Accounting and Finance, 48 (2), (pp. 279-300)

Kerby, D. y Romine, J. (2009). Develop oral presentation skills through accounting curriculum design and course-embedded assessment. Journal of Education for Business, 85 (3), (pp. 172-179)

Marambe, K.N., Vermunt, J.D. y Boshuizen, H.P.A. (2011). A cross-cultural comparison of student learning patterns in higher education. Higher Education, Vol. 64 No. 3, pp. 299316.

McCroskey, J.C. (2006). An Introduction to Rhetorical Communication (9th edition). Boston, MA: Allyn \& Bacon.

McCroskey, J.C., Burroughs, N. F., Daun, A., y Richmond, V. P. (1990). Correlates of quietness: Swedish and American perspectives. Communication Quarterly, 38, (pp. 127-137)

McCroskey, J.C. (1984). The communication apprehension perspective, in J.A. Daly and J.C. McCroskey, (EDS) Avoiding Communication: Shyness, Reticence, and Communication Apprehension, Beverly Hills, California: Sage Publications.

McCroskey, J.C. y Anderson, J.F. (1976). The relationship between communication apprehension and academic achievement among college students. Human Communication Research, 3, (pp. 73-81)

McCroskey, J.C., Daly, J.A., Richmond, V.P. y Cox, B. (1976). The effects of communication apprehension on interpersonal attraction. Human Communication Research, 2, (pp. 5165)

Michel Vázquez, G. P., Ramírez Meda, A., Arquero, J. L., y Fernández-Polvillo, C. (2017). La aprensión comunicativa en la comunicación en estudiantes de Contaduría: un estudio 
en la universidad pública en Jalisco. Revista de investigación en ciencias contables y administrativas, 2(2). (pp. 126-147)

Quible, Z. y Griffin, F. (2007). Are writing deficiencies creating a lost generation of business writers? Journal of Education for Business, 83 (1), (pp. 32-36)

Rebele, J. E., Apostolou, B. A., Buckless, F. A., Hassell, J. M., Paquette, L. R., y Stout, D. E. (1998a). Accounting education literature review (1991-1997), part I: Curriculum and instructional approaches. Journal of Accounting Education, 16(1), 1-51. DOI: 10.1016/ S0748-5751(98)00001-3

Rebele, J. E., Apostolou, B. A., Buckless, F. A., Hassell, J. M., Paquette, L. R., y Stout, D. E. (1998b). Accounting education literature review (1991-1997), part II: students, educational technology, assessment and faculty issues1. Journal of Accounting Education, 16(2), 179245. DOI: $10.1016 /$ S0748-5751(98)00010-4

Ruchala, L.V. \& Hill, J.W. (1994). Reducing accounting students' oral communication apprehension: empirical evidence. Journal of Accounting Education, 12 (4), (pp. 283-303)

Schunk, D.H. (1995). Self-efficacy and education and instruction. In J. E. Maddux (Ed.), Selfefficacy, adaptation, and adjustment: Theory, research, and application. New York: Plenum Press.

Schunk, D.H., Hanson, A.R., y Cox, P.D. (1987). Peer model attributes and children's achievement behaviors. Journal of Educational Psychology, 79, (pp. 54-61)

Sharifi, M., McCombs, G.B., Fraser, L.L. y McCabe, R.K. (2009). Structuring a competency based accounting communication course at the graduate level. Business Communication Quarterly, 72 (2), (pp.177-199)

Simons, K., Higgins, M. y Lowe, D. (1995). A profile of communication apprehension in accounting majors: implications for teaching and curriculum revision. Journal of Accounting Education, 13 (3), (pp. 299-318)

Spitzberg, B.H. y Cupach, W.R. (1984). Interpersonal Communication Competence. Beverly Hills, California: Sage Publications.

Stajkovic, A. D., y Luthans, F. (1998). Self-efficacy and work-related performance: A metaanalysis. Psychological Bulletin, 124, (pp. 240-261)

Stanga. K.G. \& Ladd, R.T. (1990). Oral communication apprehension in beginning accounting majors: an exploratory study. Issues in Accounting Education, 5(2), (pp. 180-94)

Stoner, G. y Milner, M. (2010). Embedding generic employability skills in an accounting degree: development and impediments. Accounting Education: An International Journal, 19 (1-2), (pp. 123-138)

Teven, J.J., Richmond, V. P., McCroskey, J. C., y McCroskey, L. L. (2010). Updating relationships between communication traits and communication competence. Communication Research Reports, 27, (pp. 263-270)

United Nations Conference on Trade and Development - UNCTAD (2011). Model Accounting Curriculum (Revised). Recuperado de unctad.org/en/PublicationsLibrary/ diaemisc2011d1_en.pdf

Wells, P., Gerbic, P., Kranenburg, I. y Bygrave, J. (2009). Professional skills and capabilities of accounting graduates: The New Zealand expectation gap? Accounting Education: An International Journal, 18 (4-5), (pp. 403-420) 